\title{
Preparation of PANI-PVA Composite Film with High Conductivity
}

\author{
Xiaohua Wang \\ School of Science, Chongqing Jiaotong University, Chongqing, China \\ Email: wxhcqu@126.com
}

Received 29 June 2014; revised 1 August 2014; accepted 11 September 2014

Copyright (C) 2014 by author and OALib.

This work is licensed under the Creative Commons Attribution International License (CC BY). http://creativecommons.org/licenses/by/4.0/

(c) (†) Open Access

\section{Abstract}

The PANI-PVA composite films adopting PVA as the base material and doped with hydrochloride, DBSA, amino sulfonic acid aqueous solution were prepared and their conductivity was compared. Among these PANI-PVA composite films, the conductivity of HCl-PANI-PVA film is the highest. At the same time, the influences of mass ratio between PANI and PVA, acid content, oxidant content, reaction time and drying temperature of film on the conductivity of HCl-PANI-PVA film were studied. The result shows that the conductivity of HCl-PANI-PVA film is the highest when the mass percentage of PVA is $40 \%$; the $\mathrm{C}(\mathrm{HC1})=0.5 \mathrm{~mol} / \mathrm{L}$; the $n(\mathrm{APS} / \mathrm{An})=1.0$; the reaction time is 6 hours and the drying temperature of the film is $80^{\circ} \mathrm{C}$, and that the maximum conductivity of HCl-PANI- PVA film is $16.0 \mathrm{~S} \cdot \mathrm{cm}^{-1}$.

\section{Keywords}

Polyvinyl Alcohol (PVA), Polyaniline (PANI), Hydrochloride Doping, Conductivity

Subject Areas: Composite Material, High Polymer Chemistry, Organic Polymer Materials

\section{Introduction}

PANI has the features such as favorable electrical properties, excellent environmental stability, easy preparation, monomer with low price and so on. Besides, the PANI and its modified copolymer are a low-cost raw material with the property of forming film conveniently, and a kind of a high conductive polymer material with excellent comprehensive properties. Therefore, it is regarded as one of the conducting polymers which are the most likely to realize practical application [1] [2]. At present, the development of PANI corrosion resistance coating has become a hotspot both in terms of use of conductive high polymer material and in the areas of research and development [3] [4]. The conductivity is low due to the strong interaction between PANI rigid chain in eigenstate, while $\mathrm{H}^{+}$is prone to inject in the PANI molecular chain, which increases the delocalization extent of electric charges in the molecular chain and increases the conductance in the chain. It is reported in literatures that after 
the doping of protonic acid, the conductivity will raise 12 magnitudes. After PANI doping with different acids, the conductivity of PANI film is different [5] [6]. In addition the effective composition [7] between PANI and PVA can also improve the conductivity of PANI film. The PANI-PVA composite films adopting PVA as the base material and doped with hydrochloride, DBSA, amino sulfonic acid aqueous solution were prepared and their conductivity was compared. The influences of mass ratio between PANI and PVA, acid amount, oxidant amount, reaction time and film drying temperature on the conductivity of HCl-PANI-PVA film were studied.

\section{Experiment}

\subsection{Materials and Instruments}

Polyvinyl alcohol (PVA-1799, M = 74800) was purchased from Sichuan Vinylon Plant. Aniline (An) was used after secondary distillation to colorless under reduced pressure and was purchased from Guangzhou Chemical Reagent Factory. Ammonium persulfate [( $\left(\mathrm{NH}_{4}\right)_{2} \mathrm{~S}_{2} \mathrm{O}_{8}$, APS], hydrochloride (HC1), amino sulfonic acid $\left(\mathrm{NH}_{2} \mathrm{SO}_{3} \mathrm{H}\right)$ and dodecylbenzene sulfonic acid (DBSA) were purchased from Chongqing Chuandong Chemical Reagent Factory. Epoxy resin (E-44) was purchased from Shuang Long Slurry Plant in Ping City. Boron trifluoride ethamine was purchased from Shanghai Reagent Factory. All chemical reagents used were analytically pure.

SDY-4 four point probe digital resistance meter.

\subsection{Preparation of Acid-Doped PANI-PVA Composite Films}

A certain amount of PVA was dissolved in $85^{\circ} \mathrm{C}$ water and then agitate it with electric motor for one hour. Pour the blended solution in the three-necked bottle together with $100 \mathrm{~mL} 1.0 \mathrm{~mol} / \mathrm{L}$ acid solution (hydrochloride, DBSA, amino sulfonic acid) and continue to agitate. Add a certain number of twice-reduced pressure distilled aniline after 5 minutes and then drop the $50 \mathrm{~mL}$ ammonium persulfate aqueous solution of $1 \mathrm{~mol} / \mathrm{L}$ slowly into the three-necked bottles (the period is about one hour). Put it in water bath $\left(60^{\circ} \mathrm{C}\right)$ for 6 hours with continuous agitation to produce blended film making solution. After mixing with a certain amount of epoxy resin (epoxy resin acts as the binder that can increase the adhesive force between PANI-PVA and base material) and curing agent (boron trifluoride ethylamine), cast it into film in the glass mold. Strip off the air dried film and put in a pure water for 12 hours to dissolve the foreign material on the surface of the film. Take it out and sip up with filter paper, then clamp it between two glass plates in order for vacuum drying under $60^{\circ} \mathrm{C}$. Then the PANI-PVA composite films have been prepared [8] [9].

\subsection{Characterization}

Conductivity of samples were tested in four-point probe after milled powder with a mortar and pressed into pieces [10] [11].

\section{Results and Discussion}

\subsection{Choice of Doping Acid in the PANI-PVA Composite Films}

According to the experimental procedure of Section 2.2, remain the mass ratio between PANI and PVA (the mass ratio of PVA is $30 \%$ ), and dope with hydrochloride, DBSA, amino sulfonic acid aqueous solution (the content of acid is $1.0 \mathrm{~mol} / \mathrm{L}$ ) to produce different acid-doped PANI-PVA composite films. The conductivity of different acid-doped PANI-PVA composite films is shown in Table 1. The conductivity of the HCl-PANI-PVA composite film is highest. Thanks to its strong acid of $\mathrm{HCl}$, the content of ionized $\mathrm{H}^{+}$from $\mathrm{HCl}$ is more than that of DBSA or $\mathrm{NH}_{2} \mathrm{SO}_{3} \mathrm{H}$, when the acid content is the same, which makes the doped $\mathrm{H}^{+}$content on the nitrogen atom of PANI be increased correspondingly. In order to remain electric neutrality of the composite film, the anionic quantity entering into PANI molecular chain will be increased correspondingly, which makes the conductivity of the HCl-PANI-PVA composite film be the highest. So the optimal doping acid will be HCl.

Table 1. Conductivity of different acid-doped PANI-PVA composite film.

\begin{tabular}{cccc}
\hline Composite film & HCl-PANI-PVA & DBSA-PANI-PVA & NH $_{2} \mathrm{SO}_{3} \mathrm{H}_{-} \mathrm{PANI}_{-\mathrm{PVA}}$ \\
\hline Conductivity $/\left(\mathrm{S} \cdot \mathrm{cm}^{-1}\right)$ & 13.5 & 6.2 & 1.8 \\
\hline
\end{tabular}




\subsection{Influence of PVA Content on the Conductivity of the HCl-PANI-PVA Composite Film}

Remaining other conditions unchanged, change the mass ratio between PANI and PVA to produce HCl-PANIPVA composite film with different mass percentage of PVA. The impact of PVA content on the conductivity of HCl-PANI-PVA composite film is shown in Figure 1.

It can be seen from the Figure 1 that, the conductivity increase in the beginning and then decrease with the increase of the mass percentage of PVA. If there is no PVA in the film, the conductivity is the minimum, while, with the addition of PVA, the PANI-PVA matrix has formed stable interpenetrating polymer network which increase the conductivity greatly. When the mass percentage of PVA is $40 \%$, the molecule of PANI is possible to disperse uniformly in the interpenetrating polymer network and the conductivity of HCl-PANI-PVA composite film under this ratio is the highest, and the conductivity of HCl-PANI-PVA composite film is $13.8 \mathrm{~S} \cdot \mathrm{cm}^{-1}$. However, if the PVA is in excess, the content of PANI (conductive component) in the composite film will reduce, therefore, the conductivity of HCl-PANI-PVA composite film will decrease accordingly.

\subsection{Influence of HCl Content on the Conductivity of the HCl-PANI-PVA Composite Film}

Remaining other conditions unchanged (the mass ratio of PVA is $40 \%$ ), change the amount of $\mathrm{HCl}$ to produce HCl-PANI-PVA composite film with different $\mathrm{HCl}$ content. The impact of $\mathrm{HCl}$ content on the conductivity of HCl-PANI-PVA composite film is shown in Figure 2.

It can be seen from Figure 2 that, the conductivity increase at first and then decrease with the increase of $\mathrm{HCl}$ content, it is probably owing to the ionized $\mathrm{H}^{+}$increases with the increase of $\mathrm{HCl}$ content in the system, which leads to the amount that have been doped in imine nitrogen atoms of PANI increases accordingly. Therefore, to maintain the electric neutrality, the increased anion which have been entered into PANI chain lead to the increase of conductivity of products. When the content of $\mathrm{HCl}$ is $0.5 \mathrm{~mol} / \mathrm{L}$, the maximum conductivity of $\mathrm{HCl}-$ PANI-PVA composite film is $14.6 \mathrm{~S} \cdot \mathrm{cm}^{-1}$. However, in the case of excessive acidity, the acid that hasn't participated in doping is not only difficult to be remove but also it is possible to have addition reaction with the PANI, which will destroy the large $\pi$ conjugated structure. Consequently the conductivity of HCl-PANI-PVA composite film will be decreased correspondingly [6].

\subsection{Influence of Reaction Time on the Conductivity of the HCI-PANI-PVA Composite Film}

Remaining other conditions unchanged (the mass ratio of PVA is $40 \%$, the content of $\mathrm{HCl}$ is $0.5 \mathrm{~mol} / \mathrm{L}$ ), change the reaction time to produce HCl-PANI-PVA composite film with different reaction time. The impact of reaction time on the conductivity of HCl-PANI-PVA composite film is shown in Figure 3.

It can be seen from the Figure 3 that, the conductivity increase at first and then decrease with the increase of reaction time. It is because that the PANI cannot well-polymerized and doped in the case of short response time, which leads to low conductivity. When the response time has reached 6 hours, the conductivity of HCl-PANIPVA composite film is the highest, and the conductivity of HCl-PANI-PVA composite film is $14.6 \mathrm{~S} \cdot \mathrm{cm}^{-1}$. While, in the case of excessively long response time, the aniline monomer is possible to be over oxidized, which leads to the fracture of PANI conjugated chain, and the relatively perfect doping system will be destroyed also. In addition, it may also produce insoluble PANI and lower the content of the conductive component in composite film. Consequently the conductivity of HCl-PANI-PVA composite film will decrease accordingly.

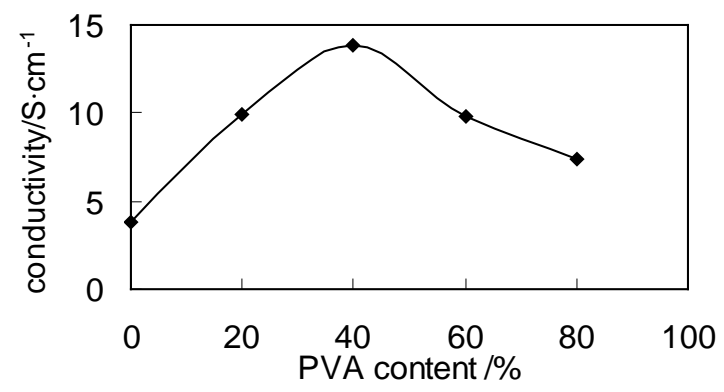

Figure 1. Influence of mass percentage of PVA on the conductivity of HCl-PANI-PVA composite film. 


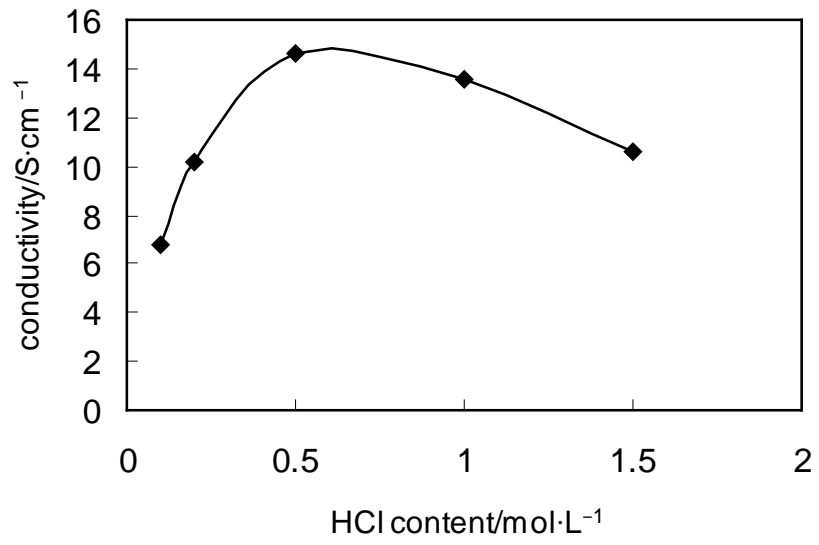

Figure 2. Influence of $\mathrm{HCl}$ content on the conductivity of $\mathrm{HCl}-$ PANI-PVA composite film.

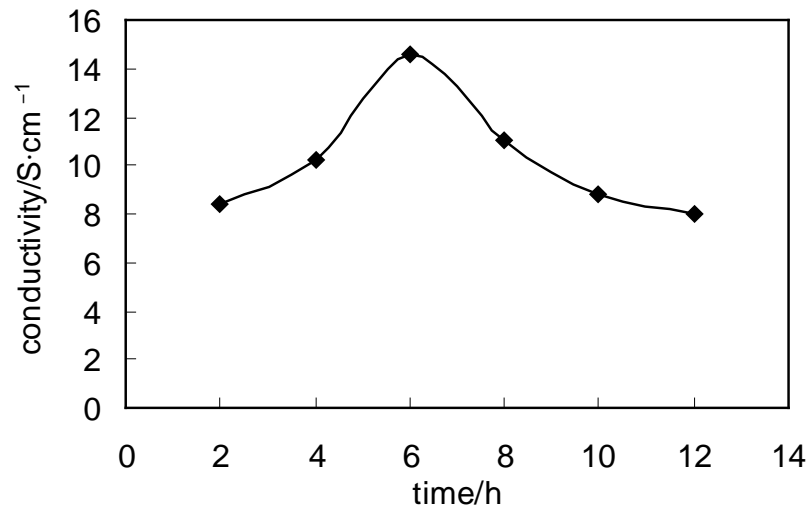

Figure 3. Influence of reaction time on the conductivity of $\mathrm{HCl}-$ PANI-PVA composite film.

\subsection{Influence of Oxidation (APS) Content on the Conductivity of the HCI-PANI-PVA Composite Film}

Remaining other conditions unchanged (the mass ratio of PVA is $40 \%$, the content of $\mathrm{HCl}$ is $0.5 \mathrm{~mol} / \mathrm{L}$, the reaction time is 6 hours), change the amount of ammonium persulfate (APS) as an oxidant to produce HClPANI-PVA composite film with different oxidation(APS) content. The impact of APS content on the conductivity of HCl-PANI-PVA composite film is shown in Figure 4.

It can be seen from Figure 4 that, the conductivity increase at first and then decrease with the increase of APS number. If the APS/An is 1.0, the conductivity of HCl-PANI-PVA composite film is the highest, and the conductivity of HCl-PANI-PVA composite film is $15.3 \mathrm{~S} \cdot \mathrm{cm}^{-1}$. However, if the initiator is in excess, there is comparatively too much active centers in the system, which is adverse to the production of PANI with high molecular weight. In addition, excess APS will over oxidize the PANI and destroy the conjugated structure in large $\pi$ electron system of the molecular chain of PANI and reduce the displacement carrier, which leads to the reduction of conductivity of HCl-PANI-PVA composite film [12].

\subsection{Influence of Drying Temperature of Film on the Conductivity of the HCl-PANI-PVA Composite Film}

Remaining other conditions unchanged (the mass ratio of PVA is $40 \%$ the mass ratio of PVA is $40 \%$, the content of $\mathrm{HCl}$ is $0.5 \mathrm{~mol} / \mathrm{L}$, the reaction time is 6 hours, the $n(\mathrm{APS} / \mathrm{An})$ is 1.0), change the drying temperature of the film to produce HCl-PANI-PVA composite film under different temperature. The impact of drying temperature of the film on the conductivity of HCl-PANI-PVA composite film is shown in Figure 5. 


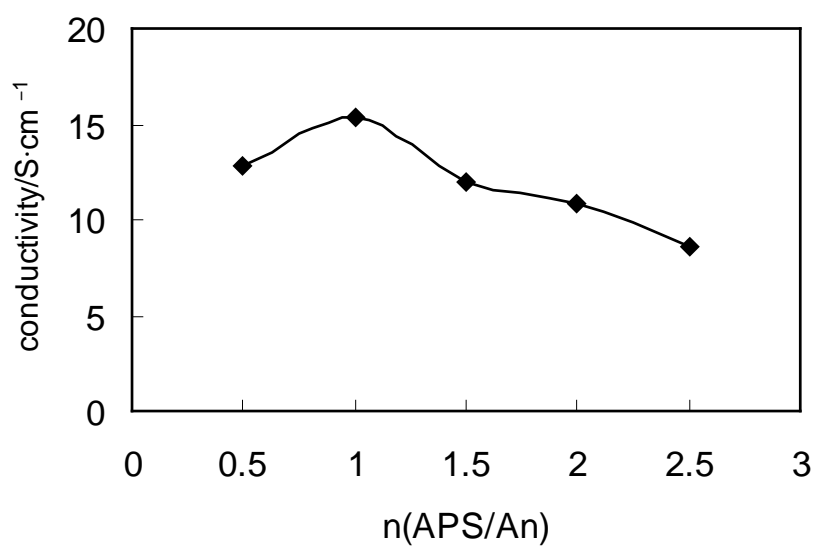

Figure 4. Influence of APS content on the conductivity of $\mathrm{HCl}-$ PANI-PVA composite film.

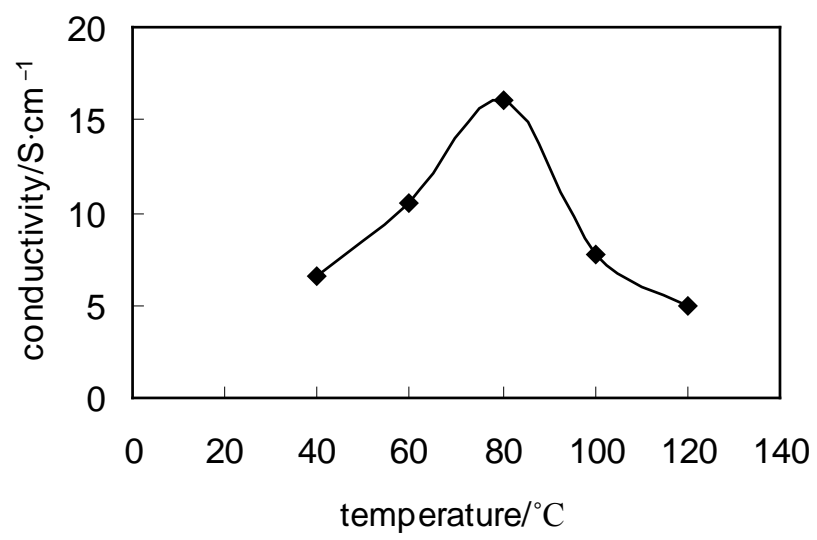

Figure 5. Influence of drying temperature of film on the conductivity of HCl-PANI-PVA composite film.

It can be seen from the Figure 5 that, the conductivity increase at first and then decrease with the increase of temperature. When the drying temperature of the film forming is $80^{\circ} \mathrm{C}$, the conductivity will reach the maximum, and the conductivity of HCl-PANI-PVA composite film is $16.0 \mathrm{~S} \cdot \mathrm{cm}^{-1}$. However, the conductivity will decrease if the temperature keeps on increasing. It seems that the overheat will generate integrated factors such as local over oxidation, increase of by-products, shorter induction period, reduction of molecular weight of PANI and the conjugation extent so on [13].

\section{Conclusions}

The PANI-PVA composite films adopting PVA as the base material and doped with hydrochloride, DBSA, amino sulfonic acid aqueous solution were prepared and their conductivity was compared. Among these PANIPVA composite films, the conductivity of HCl-PANI-PVA film is the highest. The reason is that, as for the same acid concentration, the number of ionized $\mathrm{H}^{+}$of hydrochloric acid is greater than that of ionized $\mathrm{H}^{+}$of DBSA and $\mathrm{NH}_{2} \mathrm{SO}_{3} \mathrm{H}$ due to strong acidity of hydrochloric acid, and there is a comparatively greater number of $\mathrm{H}^{+}$that has doped with the imine nitrogen atoms in the PANI chain. To maintain the electric neutrality, there is a relatively large amount of anion entering in the PANI chain.

At the same time, the influences of mass ratio between PANI and PVA, acid content, oxidant content, reaction time and drying temperature of the film on the conductivity of HCl-PANI-PVA film were studied. The result shows that the conductivity of HCl-PANI-PVA film is the highest when the mass percentage of PVA is $40 \%$; the $\mathrm{C}(\mathrm{HC} 1)=0.5 \mathrm{~mol} / \mathrm{L}$; the $n(\mathrm{APS} / \mathrm{An})=1.0$; the reaction time is 6 hours and the drying temperature of the film is $80^{\circ} \mathrm{C}$, and that the maximum conductivity of HCl-PANI-PVA film is $16.0 \mathrm{~S} \cdot \mathrm{cm}^{-1}$. 


\section{References}

[1] Ding, Y. and Yang, J.P. (2008) Emulsion Polymerization Process Research of Phenylamine in DBSA/Water Systems. Acta Polymerica Sinica, 3, 246-250. http://dx.doi.org/10.3724/SP.J.1105.2008.00246

[2] Liu, L., Zhong, F.C. and Wang, X.C. (2008) In-Situ Emulsion Polymerization Polyaniline PANI/PMMA and Its Characterization. Chemical Industry and Engineering Progress, 27, 1230-1233.

[3] Zhang, J.Y., Li, J. and Wang, X.H. (2005) The Application of PANI in the Corrosion Prevention Field. Journal of Functional Polymers, 12, 350-356.

[4] Zu, X.H., Hu, J.Q. and Wang, F. (2008) Research and Development of Environmental Friendly Antirust Coatings. Chemical Industry and Engineering Progress, 27, 1394-1399.

[5] Yan, H.Y., Hu, Z.Y. and Ku, K.C. (2005) Research of Conductivity and Spectral Features of Function Sulfonic Acid Doping. Journal of Materials Engineering, 1, 50-52, 57.

[6] Kuang, Y.Z. (2004) Synthesis and Application of Intrinsically Conductive Polymers. Fine Chemical Intermediates, 34, 18-20.

[7] Cho, M.S., Park, S.Y., Hwang, J.Y., et al. (2004) Synthesis and Electrical Properties of Polymer Coatings with Polyaniline Nanoparticles. Materials Science and Engineering: C, 24, 15-18.

[8] Kuang, T., Liao, L.F. and Zhou, X. (2006) Study of Preparation of PANI-PVA Conductive Blend Membrane. Applied Chemical Industry, 35, 900-902.

[9] Zhong, P. and Huang, J.J. (2009) The Development and Performance Test of the PANI/PVA. Fiber Reinforced Plastics/Composites, 1, 58-61.

[10] Ma, L., Feng, L.J. and Gan, M.Y. (2008) Synthesis and Performance Study on PANI with Complex Acid Doping. Chinese Journal of Applied Chemistry, 25, 142-146.

[11] Sun, Y.C. (1990) Semiconductor Testing Technology. Metallurgical Industry Publishers, Beijing, in Press.

[12] Fu, H.Q., Zhang, X.Y. and Huang, H. (2005) Emulsion Polymerization Method for Perparing Polyaniline and Its Performance. CIESC Journal, 56, 1790-1793.

[13] Ren, B. and Yu, C. (2005) Synthesize and Properties of Conductive Polyaniline. Chinese Journal of Spectroscopy Laboratory, 2, 148-151. 